\title{
Diffractive production of heavy mesons at the LHC
}

\author{
Marta Łuszczak ${ }^{1, \star}$ and Antoni Szczurek ${ }^{1,2}$ \\ ${ }^{1}$ University of Rzeszów, PL-35-959 Rzeszów, Poland \\ ${ }^{2}$ Institute of Nuclear Physics, Polish Academy of Sciences, Kraków, Poland
}

\begin{abstract}
We discuss diffractive production of heavy mesons at the LHC. In addition to standard collinear approach, for a first time we propose a $k_{t}$-factorization approach to the diffractive processes. The unintegrated (transverse momentum dependent) diffractive parton distributions in proton are calculated with the help of the Kimber-Martin-Ryskin prescription where collinear diffractive PDFs are used as input. Some correlation observables, like azimuthal angle correlation between $c$ and $\bar{c}$, and $c \bar{c}$ pair transverse momentum distribution were obtained for the first time. The results of the new approach are compared with those of the standard collinear one. Significantly larger cross sections are obtained in the $k_{t}$-factorization approach where some part of higher-order effects is effectively included.
\end{abstract}

\section{Introduction}

Diffractive hadronic processes were studied theoretically in the so-called resolved pomeron model [1]. This model, previously used to describe deep-inelastic diffractive processes must be corrected for absorption effects related to hadron-hadron interactions. In theoretical models this effect is taken into account approximately by multiplying the diffractive cross section calculated using HERA diffractive PDFs by a kinematics independent factor called the gap survival probability $-S_{G}$. Two theoretical groups specialize in calculating such probabilities $[2,3]$.

In this study we consider diffractive production of charm for which rather large cross section at the LHC are expected, even within the leading-order (LO) collinear approach [4]. On the other hand, it was shown that for the inclusive non-diffractive charm production the LO collinear approach is a rather poor approximation and higher-order corrections are crucial. Contrary, the $k_{t}$-factorization approach, which effectively includes higher-order effects, gives a good description of the LHC data for inclusive charm production at $\sqrt{s}=7 \mathrm{TeV}$ (see $e . g$. Ref. [5]). This strongly suggests that application of the $k_{t}$-factorization approach to diffractive charm production is useful. This presentation is based on our recent study presented in [6]. Here we present only results at the quark/antiquark level.

\section{Formalism}

A sketch of the theoretical formalism is shown in Fig. 1. Here, extension of the standard resolved pomeron model based on the LO collinear approach by adopting a framework of the $k_{t}$-factorization is proposed as an effective way to include higher-order corrections.

\footnotetext{
^e-mail: luszczak@ur.edu.pl,
} 

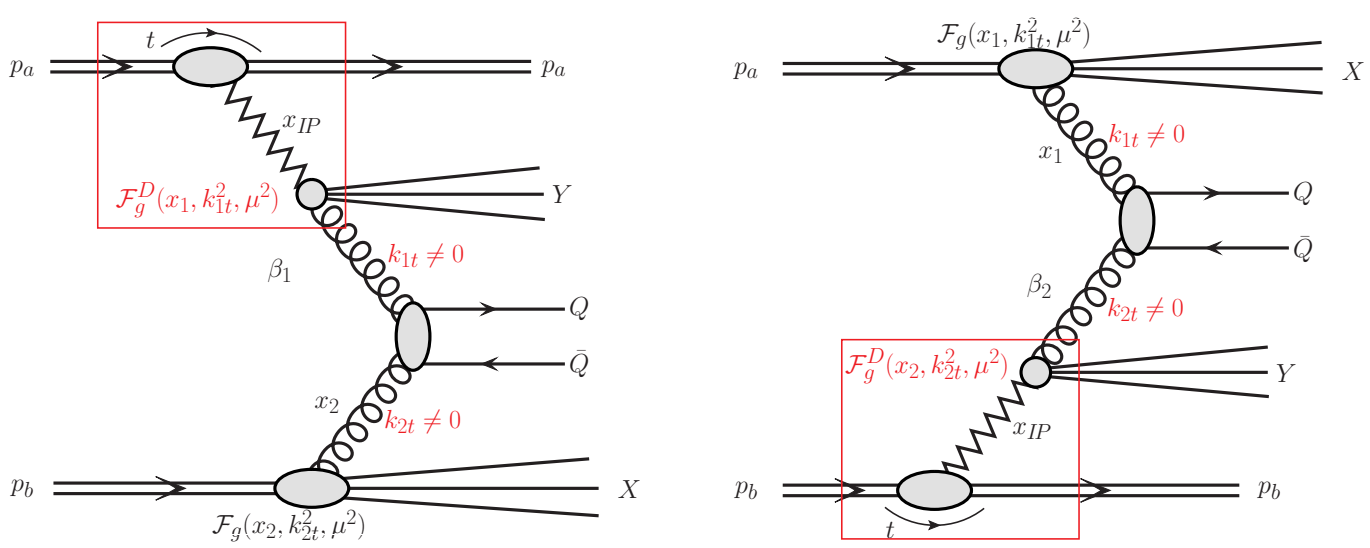

Figure 1. A diagrammatic representation for single-diffractive production of heavy quark pairs within the $k_{t^{-}}$ factorization resolved pomeron approach.

Details of our new calculations can be found in Ref. [6].

\section{Results}

In Fig. 2 we show rapidity (left panel) and transverse momentum (right panel) distribution of $c$ quarks (antiquarks) for single diffractive production at $\sqrt{s}=13 \mathrm{TeV}$. Distributions calculated within the LO collinear factorization (black long-dashed lines) and for the $k_{t}$-factorization approach (red solid lines) are shown separately. We see significant differences between the both approaches, that are consistent with the conclusions from similar studies of standard non-diffractive charm production (see e.g. Ref. [5]). Here we confirm that the higher-order corrections are very important also for the diffractive production of charm quarks.
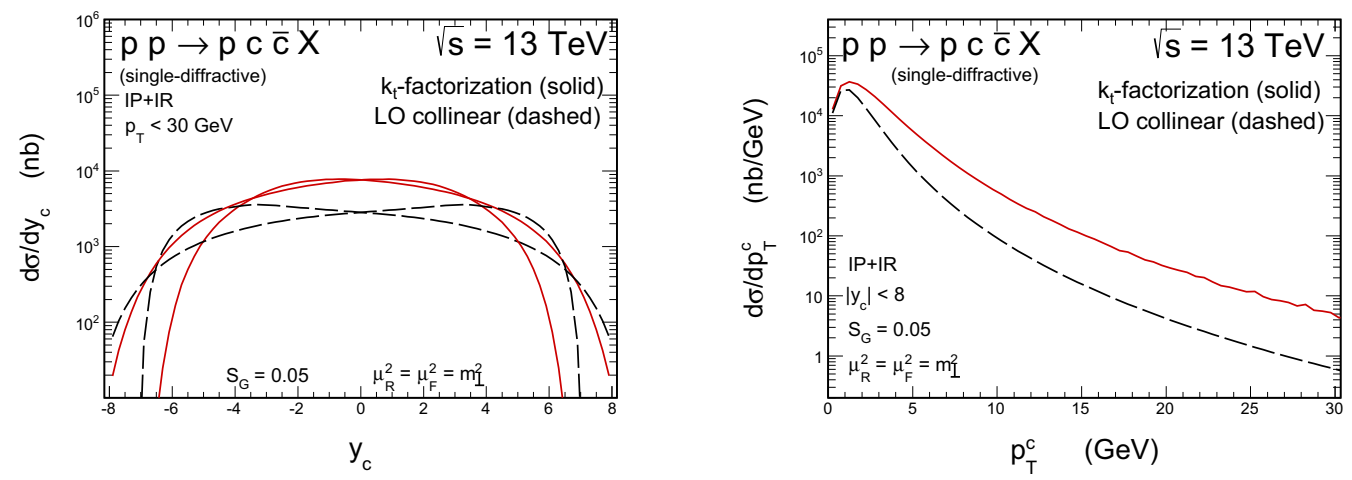

Figure 2. Rapidity (left panel) and transverse momentum (right panel) distributions of $c$ quarks (antiquarks) for a single-diffractive production at $\sqrt{s}=13 \mathrm{TeV}$. Components of the $g(I P)-g(p), g(p)-g(I P), g(I R)-g(p)$, $g(p)-g(\mathbb{R})$ mechanisms are shown. 
The correlation observables cannot be calculated within the LO collinear factorization but can be directly obtained in the $k_{t}$-factorization approach. The distribution of azimuthal angle $\varphi_{c \bar{c}}$ between $c$ quarks and $\bar{c}$ antiquarks is shown in the left panel of Fig. 3. The $c \bar{c}$ pair transverse momentum distribution $p_{T}^{c \bar{c}}=\left|\overrightarrow{p_{t}^{c}}+\overrightarrow{p_{t}^{c}}\right|$ is shown in the right panel. Results of the full phase-space calculations illustrate that the quarks and antiquarks in the $c \bar{c}$ pair are almost uncorrelated in the azimuthal angle between them and are often produced in the configuration with quite large pair transverse momenta.
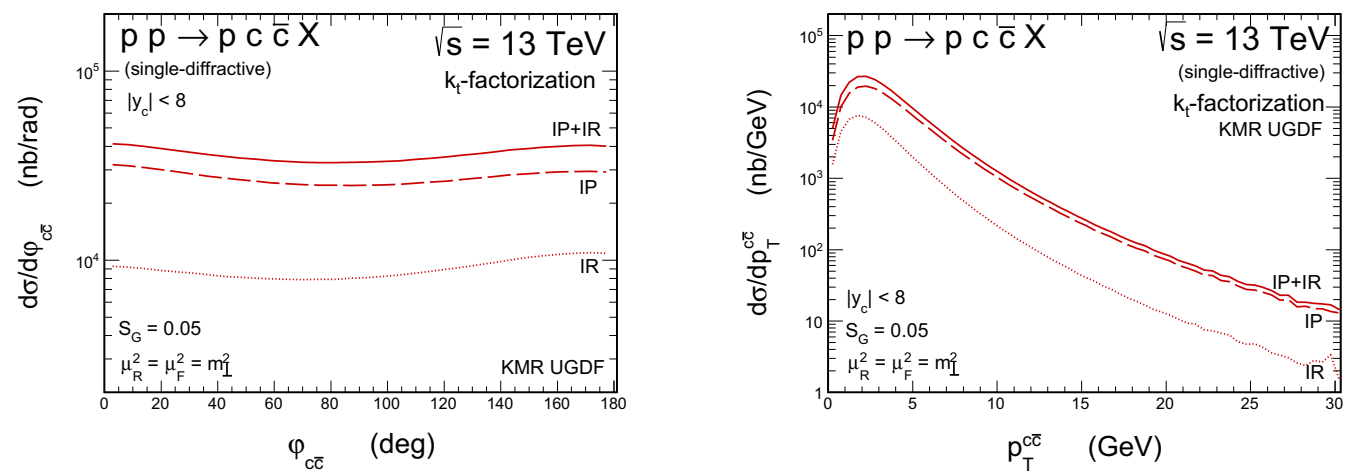

Figure 3. The distribution in $\phi_{c \bar{c}}$ (left panel) and distribution in $p_{T}^{c \bar{c}}$ (right panel) in the $k_{t}$-factorization approach at $\sqrt{s}=13 \mathrm{TeV}$.
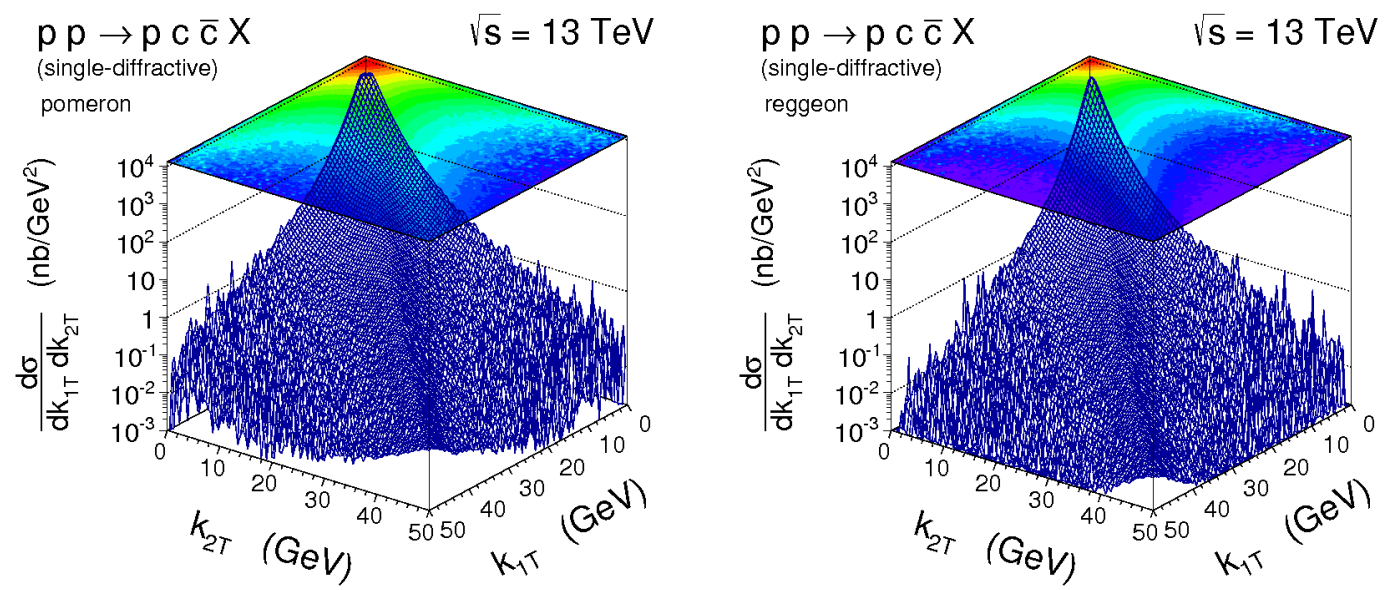

Figure 4. Double differential cross sections as a function of initial gluons transverse momenta $k_{1 T}$ and $k_{2 T}$ for single-diffractive production of charm at $\sqrt{s}=13 \mathrm{TeV}$. The left and right panels correspond to the pomeron and reggeon exchange mechanisms, respectively.

Figures 4 and 5 show the double differential cross sections as a functions of transverse momenta of incoming gluons $\left(k_{1 T}\right.$ and $\left.k_{2 T}\right)$ and transverse momenta of outgoing $c$ and $\bar{c}$ quarks $\left(p_{1 T}\right.$ and $\left.p_{2 T}\right)$, respectively. We observe quite large incident gluon transverse momenta. The major part of the cross 

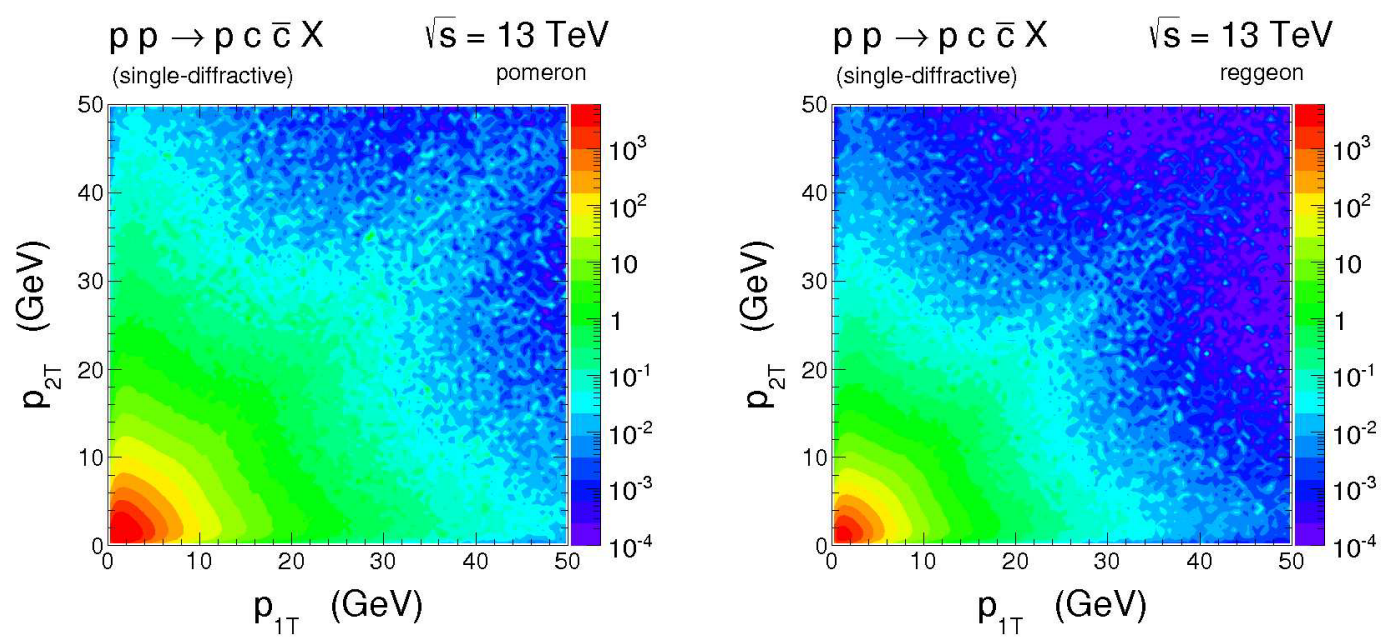

Figure 5. Double differential cross sections as a function of transverse momenta of outgoing $c$ quark $p_{1 T}$ and outgoing $\bar{c}$ antiquark $p_{2 T}$ for single-diffractive production of charm at $\sqrt{s}=13 \mathrm{TeV}$. The left and right panels correspond to the pomeron and reggeon exchange mechanisms, respectively.

section is concentrated in the region of small $k_{t}$ 's of both gluons but long tails are present. Transverse momenta of the outgoing particles are not balanced as they were in the case of the LO collinear approximation.

\section{Conclusions}

Charm production is a good example where the higher-order effects are very important. For the inclusive charm production we have shown that these effects can be effectively included in the $k_{t^{-}}$ factorization approach [5]. In our approach we decided to use the so-called KMR method to calculate unintegrated diffractive gluon distribution (UDGD). Having obtained the UDGD we have performed calculations of several single-particle and correlation distributions. In general, the $k_{t}$-factorization approach leads to larger cross sections. However, the $K$-factor is strongly dependent on phase space point. Some correlation observables, like azimuthal angle correlation between $c$ and $\bar{c}$, and $c \bar{c}$ pair transverse momentum distributions were obtained in [6] for the first time.

\section{Acknowledgement}

This work was partially supported by the Polish National Science Centre grant DEC-2013/09/D/ST2/03724 as well as by the Centre for Innovation and Transfer of Natural Sciences and Engineering Knowledge in Rzeszów.

\section{References}

[1] G. Ingelman, P.E. Schlein, Phys. Lett. B 152, 256 (1985)

[2] V. A. Khoze, A. D. Martin and M. G. Ryskin, Eur. Phys. J. C 18, 167 (2000)

[3] U. Maor, AIP Conf. Proc. 1105, 248 (2009)

[4] M. Luszczak, R. Maciula and A. Szczurek, Phys. Rev. D 91, 054024 (2015) 
[5] R. Maciula and A. Szczurek, Phys. Rev. D 87, 094022 (2013)

[6] M. Luszczak, R. Maciula, A. Szczurek and M. Trzebinski, arXiv:1606.06528 [hep-ph] 